\title{
Multinucleate Cell Angiohistiocytoma: A Case Report and Unique Treatment Consideration
}

\author{
Maria Karim, BA ${ }^{1}$, Marlyn Wu, $\mathrm{DO}^{2}$, Adriana Ros, $\mathrm{DO}^{3}$ \\ ${ }^{1}$ Hackensack University Medical Center, Hackensack, NJ \\ ${ }^{2}$ Hackensack Meridian Health Palisades Medical Center, North Bergen, NJ \\ ${ }^{3}$ Dermatology Institute \& Laser Center, Clifton, NJ
}

\section{ABSTRACT}

Introduction: Multinucleate cell angiohistiocytoma (MCAH) is a benign, yet exceedingly rare vascular and fibrohistiocytic proliferation. The lesions are typically asymptomatic and are commonly cosmetically displeasing to patients. ${ }^{1}$ Previous reports have demonstrated success in treating MCAH with fractional ablative $\mathrm{CO}_{2}$ laser, argon laser, pulsed dye laser, and KTP lasers. ${ }^{3}$ The scarcity of reported cases may suggest that the lesion is commonly misdiagnosed, and we report this case to highlight the characteristic features of MCAH with successful treatment with Neodymium YAG laser.

Case Presentation: A 64-year- old female presented to our clinic with a 1-month history of multiple erythematous to violaceous lesions on her bilateral hands. The lesions were asymptomatic, but cosmetically displeasing for the patient. Past medical history and family history was noncontributory. Examination revealed several 2-6 mm well- circumscribed, erythematous, indurated smooth papules on the bilateral dorsal and in the second web space of the left hand. Shave biopsy of the lesions were consistent with $\mathrm{MCAH}$. She was initially treated with topical steroids twice daily for 2 weeks, with no improvement. The patient received 3 treatments with Neodymium YAG, 4 weeks apart. This treatment was discontinued after 3 sessions due to the dramatic visible improvements in regards to induration and discoloration of the lesions.

Conclusion: MCAH is an extremely uncommon lesion, diagnosis is made on the basis of clinical examination, histopathologic and immunohistochemical analysis of the lesions. This case illustrates diagnosis and successful treatment of MCAH with Neodymium YAG and offers a unique treatment consideration for patients.

\section{INTRODUCTION}

Multinucleate cell angiohistiocytoma (MCAH) is a benign, yet exceedingly rare vascular and fibrohistiocytic proliferation. MCAH is characterized by multiple redbrown or violaceous papules measuring 2$15 \mathrm{~mm}$ in diameter typically occurring on the face and distal extremities, most commonly in middle- aged and elderly females. ${ }^{1}$ Lesions are typically characterized by multinucleated giant cells with angulated cytoplasm, dilated capillaries and small vessel proliferation in the papillary to mid dermis, and thickened collagen bundles with fibrous stroma. ${ }^{2}$ The lesions are typically asymptomatic, but may be associated with mild pruritus, and are commonly cosmetically displeasing to patients. ${ }^{3}$

Previous reports have demonstrated success in treating MCAH with fractional ablative $\mathrm{CO}_{2}$ laser, argon laser, pulsed dye laser, intense pulsed light, and KTP lasers. ${ }^{2,4,5,6,7}$ The scarcity of reported cases 
may suggest that the lesion is commonly misdiagnosed. We report this case to highlight the characteristic features of $\mathrm{MCAH}$, and to report a unique case of successful treatment of MCAH with neodymium (nd) YAG laser.

\section{CASE REPORT}

A 64-year- old female presented to our clinic with a 1- month history of multiple erythematous to violaceous lesions on her bilateral hands. The lesions were asymptomatic, but cosmetically displeasing for the patient. Medical history was significant for hypercholesterolemia and hyperthyroidism. Family history of similar lesions was noncontributory. A routine investigation including blood count and metabolic profile did not reveal any underlying abnormality.

Physical examination revealed several discrete 2-6 mm, well- circumscribed, erythematous, indurated smooth papules on the dorsal hands bilaterally, and in the second web space of the left hand (Fig 1).

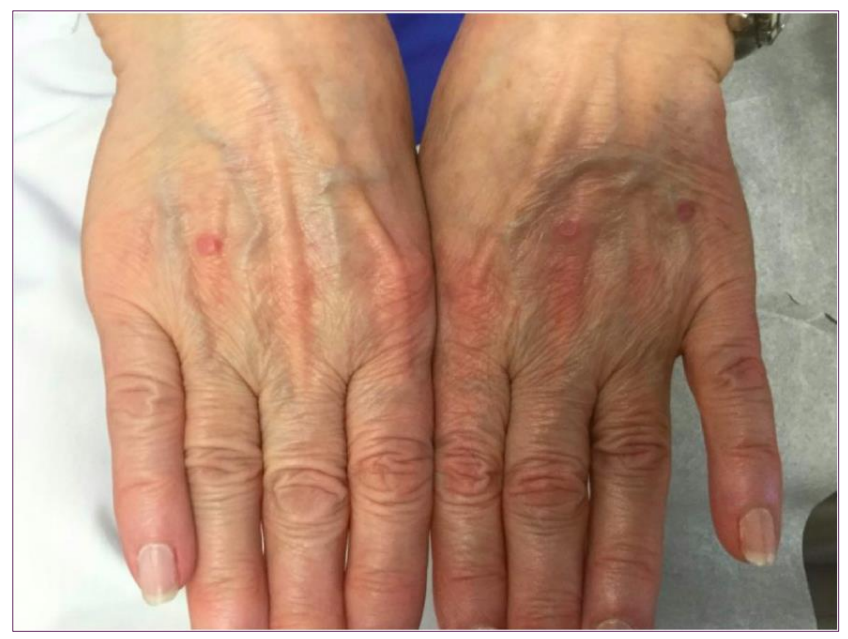

Figure 1. Multiple, well- circumscribed, erythematous, indurated smooth papules measuring 2-6 $\mathrm{mm}$ each, on the dorsal hands bilaterally, and in the second web space of the left hand
The differential diagnosis included Gottron papules, papular granuloma annulare and lichen planus. Shave biopsy of the lesions revealed an increased number of dilated blood vessels, multinucleated cells, and thickened collagen bundles. These findings are consistent with MCAH (Fig 2). She was initially treated with topical Betamethasone dipropionate $0.05 \%$ cream twice daily for 2 weeks previously, with no improvement in the gross appearance of the lesions.

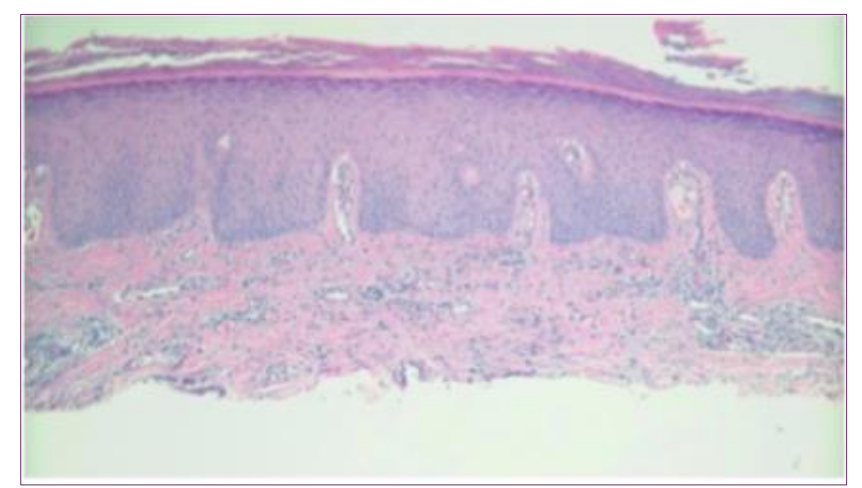

Figure 2. The pathologic examination revealed an increased number of dilated blood vessels, multinucleated cells, and thickened collagen bundles. These findings are consistent with MCAH (Hematoxylin-eosin stain).

The patient received treatment with $\mathrm{Nd}$ : YAG laser (wavelength 1064 nd yag, fluence: level $6(6 \mathrm{~mm})$, level $8(2 \mathrm{~mm})$, pulse duration: 6 microsecs, spot size: $6 \mathrm{~mm}$, $2 \mathrm{~mm}$ ), each session 4 weeks apart. After 3 sessions, the patient had dramatic visible improvement in the induration and discoloration of the lesions. (Fig 3)

\section{DISCUSSION}

Multinucleate cell angiohistiocytoma is an extremely uncommon clinical entity, with relatively few cases reported since it was first described in 1985 by Smith and Wilson Jones. ${ }^{8}$ The clinical presentation of MCAH involves one or several firm, erythematous 
to violaceous dome- shaped papules with a flat top, usually presenting on the distal

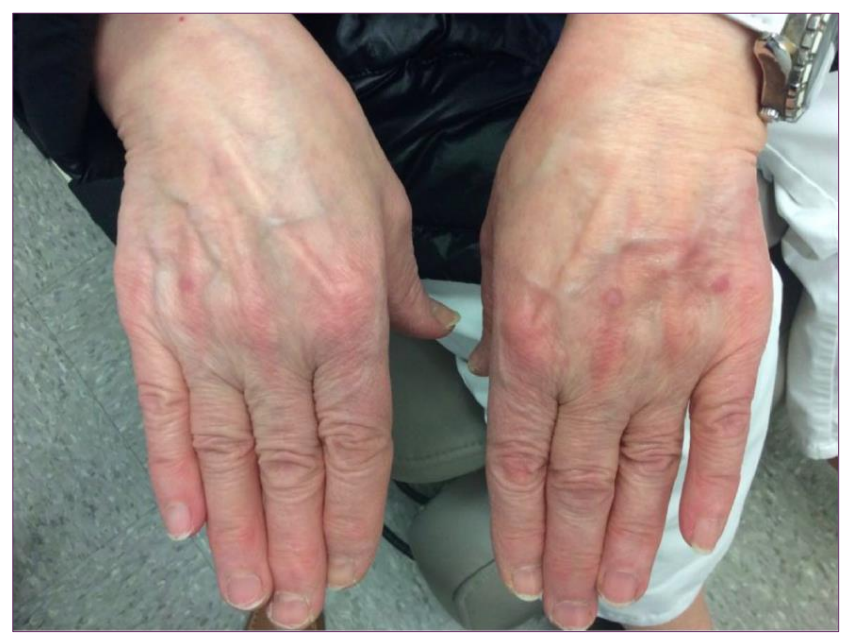

Figure 3. Post 3 sessions of Neodymium YAG laser treatments.

extremities. The lesions tend to be welldemarcated and follow a random, linear, or annular distribution pattern. The lesions tend to follow a slowly progressive course, and rarely remit spontaneously. ${ }^{9}$

Diagnosis is made on the basis of clinical examination and histopathologic and immunohistochemical analysis of the lesions. Histopathologically, characteristic findings include basophilic, stellate multinucleated cells, small vessel inflammation and proliferation, dermal fibrosis, and sparse lymphohistiocytic infiltrates. The multinucleate cells characteristically have angulated cytoplasms, with several hyperchromatic nuclei arranged around the periphery of the cells, comparable to the multinucleated cells seen in dermatofibroma. ${ }^{9}$

Immunohistochemical staining has been found to be positive for vimentin, CD34, CD31, and factor VIII- related antigen, with some cases reported to have CD68 expression of multinucleate cells depending on varying maturation or degenerative stages of the cells. ${ }^{9}$ This staining pattern suggests a fibroblastic origin preceding the multinucleate cells, rather than a monocytic one due to the lack of expression of monocyte markers.

Several hypotheses have been proposed to contribute to the progression of the lesions, though the exact pathogenic mechanism has yet to be elucidated. The role of mast cell degranulation, and more specifically, the release of interleukin- 4, have been implicated in the pathogenesis of the disease. ${ }^{10}$ Cesinaro et al reported a potential link to female hormones, elucidating the relationship between lesions expressing estrogen receptor alpha on their interstitial and multinucleate cells, and the female predominance associated with the disease. ${ }^{11}$

MCAH tends to have a slowly progressive and indolent, yet benign course. To date, extracutaneous symptoms and malignant transformation have not been reported, while spontaneous regression has been. ${ }^{10}$ These findings together suggest a reactive inflammatory, rather than neoplastic, process. ${ }^{3}$ Accordingly, it is important to biopsy the lesions to correctly diagnose and appropriately treat patients. It is crucial to recognize that $\mathrm{MCAH}$ may present as single or multiple papules, with a distribution that may imitate that of an inflammatory process. $^{1}$

\section{CONCLUSION}

Although there is no consensus on the optimal treatment regimen, management of the lesions typically begins with topical or intralesional corticosteroids. ${ }^{2}$ Successful treatment has been reported using surgical excision, cryotherapy, pulsed- dye or argon laser, intense pulsed light, or carbon dioxide laser. ${ }^{2}$ Further studies are needed to 
compare the efficacy of various treatment modalities in patients with MCAH.

\section{Conflict of Interest Disclosures: None}

Funding: None

\section{Corresponding Author:}

Marlyn Wu, DO

7650 River Road Suite 120

North Bergen, NJ 07047

Phone: 347-669-4034

Email: Drwuderm@gmail.com

\section{References:}

1. Frew J. W. (2015). Multinucleate cell angiohistiocytoma: clinicopathological correlation of 142 cases with insights into etiology and pathogenesis. The American Journal of dermatopathology, 37(3), 222228.

2. Tuchayi, S. M., Garibyan, L., \& Lee, K. C. (2019). Successful treatment of multinucleate cell angiohistiocytoma with fractionated ablative $\mathrm{CO} 2$ laser. JAAD Case Reports, 5(4), 297-299.

3. Wang, M., Abdul-fattah, B., Wang, C., Zhao, Y., Qu, X., Al-Muriesh, M., ... \& Chen, S. (2017). Generalized multinucleate cell angiohistiocytoma: case report and literature review. Journal of cutaneous pathology, 44(2), 125-134.

4. Edgerton, B., Ladha, M. A., Hunter, C., Devani, A. R., \& Prajapati, V. H. (2019). Successful treatment of multinucleate cell angiohistiocytoma in an adult male patient with potassium-titanyl-phosphate laser in combination with intralesional corticosteroids. JAAD Case Reports, 5(10), 880-882.

5. Kopera, D., Smolle, J., \& Kerl, H. (1995). Multinucleate cell angiohistiocytoma: treatment with argon laser. The British journal of dermatology, 133(2), 308-310.

6. Richer, V., \& Lui, H. (2016). Facial multinucleate cell angiohistiocytoma: longterm remission with $585 \mathrm{~nm}$ pulsed dye laser. Clinical and experimental dermatology, 41(3), 312-313.

7. Fernández-Jorge, B., del Pozo, J., GarcíaSilva, J., Barja, J. M., Yebra-Pimentel, M. T., \& Fonseca, E. (2009). Multinucleate cell angiohistiocytoma: treatment using intense pulsed light. Dermatologic surgery : official publication for American Society for Dermatologic Surgery [et al.], 35(7), 11411143.

8. Smith, N.P. and Jones, E.W. (1985), Multinucleate cell angiohistiocytoma-a new entity. British Journal of Dermatology, 113: 15-15.

9. Applebaum, D. S., Shuja, F., Hicks, L., Cockerell, C., \& Hsu, S. (2014). Multinucleate cell angiohistiocytoma: a case report and review of the literature. Dermatology online journal, 20(5).

10. Roy, S. F., Dong, D., Myung, P., \& McNiff, J. M. (2019). Multinucleate cell angiohistiocytoma: a clinicopathologic study of 62 cases and proposed diagnostic criteria. Journal of cutaneous pathology, 46(8), 563-569.

11. Cesinaro, A. M., Roncati, L., \& Maiorana, A. (2010). Estrogen receptor alpha overexpression in multinucleate cell angiohistiocytoma: new insights into the pathogenesis of a reactive process. The American Journal of dermatopathology, 32(7), 655-659. 